\title{
Intracytoplasmic sperm injection with fresh versus cryopreserved testicular sperm in azoospermic patients
}

Kani M. Falahid

\begin{abstract}
Background: The purpose of this study is to compare the outcome of intracytoplasmic sperm injection (ICSI) using fresh sperm versus frozen-thawed sperm in both obstructed and non-obstructed azoospermias. This retrospective study included 159 ICSI cycles from 126 couples. In 91 obstructed azoospermia cases, 66 cycles were treated with fresh testicular sperm and 25 cycles were treated with frozen-thawed testicular samples. In 68 non-obstructed azoospermia cases, 32 cycles were treated with fresh testicular sperm and 36 cycles were treated with frozenthawed testicular sperm, and the main measure and outcomes calculated are fertilization rate, clinical pregnancy, and live birth rate.
\end{abstract}

Results: In case of obstructed azoospermia, there were no statistically significant differences between fresh sperm and frozen-thawed testicular sperm used for ICSI regarding fertilization rate, clinical pregnancy rate, and live birth rate as shown (57\%, 47\%, $0.093 p$ value; $23.7 \%, 17.4 \%, 0.54 p$ value; and $11.9 \%, 8.7 \%, 0.68 p$ value, respectively). Nonobstructed azoospermia cases also show no significant differences in fertilization rate (37\%, 36\%, $0.91 p$ value), clinical pregnancy rate (20\%, 14.3\%, $0.58 p$ value), and live birth rate (4\%, 3.6\%, $0.93 p$ value).

Conclusion: Cryopreservation of testicular sperm is reliable if carried out before ovulation induction especially in cases with non-obstructive azoospermia

Keywords: Obstructive azoospermia, Non-obstructive azoospermia, ICSI, Fresh testicular sperm, Frozen-thawed testicular sperm

\section{Background}

Intracytoplasmic injection with testicular sperm since its introduction in 1993 [1, 2] has become a routine treatment procedure for patients with azoospermia, whether they suffer from obstructive azoospermia $(\mathrm{OA})$, which is defined as the absence of spermatozoa in the ejaculate despite normal spermatogenesis (OA is a common urologic condition and accounts for $6.1 \%$ [1] to $13.6 \%$ [2] of patients presenting for fertility evaluation $[3,4])$, or nonobstructive azoospermia (NOA) which is defined as no sperm in the ejaculate due to failure of spermatogenesis and is the most severe form of male infertility. Testicular failure affects approximately $1 \%$ of the male population and $10 \%$ of men who seek fertility evaluation [5].
Correspondence: Kani.raza@univsul.edu.iq

College of Medicine, University of Sulaimani, Sulaymaniyah City, KRG, Iraq
High fertilization rates, pregnancy rates, and implantation rates are obtained in obstructive patients [6-8].

In the population of patients with NOA, however, the probability of finding sperm is only $50 \%$ in a nonselected population [9], but fertilization and pregnancy rates can reach acceptable levels [9-13].

Surgically retrieved spermatozoa from azoospermic patient are a routine procedure used for ICSI in IVF centers. However, the recovery of fresh testicular biopsies at the day of oocyte retrieval is highly stressful for the couple as it implies a $50 \%$ risk of pointless ovarian stimulation of the female partner. Moreover, repeated testicular surgery in subsequent ICSI cycles may cause testicular devascularization and possibly permanent injury $[14,15]$.

Several centers initiated the use of frozen-thawed surgically retrieved spermatozoa in addition to freshly retrieved spermatozoa to achieve ICSI pregnancy, and the 
outcome of cycles using fresh or frozen-thawed retrieved spermatozoa is the source of much debate. The majority or reports suggest no significant worsening in outcome with the use of cryopreserved gametes after surgical retrieval $[13,16-18]$. Others, however, have reported a significantly lower FR $[19,20]$, clinical pregnancy rate (CPR) [10, 20], and implantation rate [10, 21] using cryopreserved spermatozoa.

The aim of our study is to present our data on ICSI cycles using surgically retrieved sperm from azoospermic men, to assess embryo development, and to compare the outcomes of ICSI for fresh sperm versus frozen-thawed testicular sperm in both OA and NOA.

\section{Methods}

This retrospective study included 159 ICSI cycles from 126 couples, with 98 cycles treated with fresh testicular sperm and 61 cycles treated with frozen-thawed testicular samples. Before assisted reproduction, 126 males were evaluated by clinical history, physical examination, and hormonal assay. Female partners were also assessed by full history and infertility investigation, and informed consent was taken from all patients.

The etiology of azoospermia was classified as obstructive azoospermia OA (91 cycles) and non-obstructive azoospermia NOA (68 cycles).

In all cases, surgical sperm was retrieved by the surgeon, and either testicular sperm aspiration under local anesthesia or testicular sperm extraction under general anesthesia was done for them.

Testicular specimens placed in petri dish with HEPESbuffered culture medium were processed by mechanical shredding [22], and the suspension was explored under an inverted microscope at $\times 400$ magnification, when spermatozoa found in specimens was used either freshly for ICSI or when done for diagnostic specimens cryopreserved for next cycle.

\section{Ovarian stimulation and oocyte retrieval}

In all patients, short agonist protocols were used to stimulate follicular development. The short protocol includes pituitary desensitization with gonadotropinreleasing hormone agonist (Decapeptyl $0.1 \mathrm{mg}$, Ferring $\mathrm{GmbH}$, Germany) at day 2 of the cycle and ovarian stimulation with follicle-stimulating hormone (fostimon, IBSA, Lugano 3, Suisse; Gonal-f, Laboroteries Serono S.A, Switzerland; Puregon, Schering-Plough, NV Organon, Oss, Netherlands) or combined LH and FSH (Merional, IBSA, Lugano 3, Suisse; Menegon, Ferring $\mathrm{GmbH}$, Germany) starting at day 3 of the cycle. Measuring serum E2 and performing transvaginal ultrasound monitored the follicular development. Ovulation and final maturation of the ova were induced with human chorionic gonadotropin (hCG) (Choriomon, IBSA,
Lugano 3, Suisse) as a single dose of 10,000 IU, when the leading follicle reached $18 \mathrm{~mm}$ in average diameter in addition to the presence of at least two other follicles of more than $16 \mathrm{~mm}$ in size and E2 $>500 \mathrm{pg} / \mathrm{ml}$, then the oocytes were retrieved. Oocytes were aspirated $34-36 \mathrm{~h}$ after hCG administration. Oocyte retrieval was performed by transvaginal ultrasound-guided puncture using 16-gage, $35-\mathrm{cm}$ double lumen aspiration needle (William A. Cook, Australia Pty Ltd.) with a negative pressure of $20 \mathrm{mmHg}$.

\section{Oocytes denudation and evaluation}

Removal of the surrounding cumulus cells was accomplished by a combined enzymatic and mechanical treatment carried out under a stereoscopic dissecting microscope. Oocytes were denudated from cumulus oophorus by exposure to $80 \mathrm{IU} / \mathrm{ml}$ hyaluronidase enzyme in HEPES-buffered medium (Hyase, FertiPro N.V., Belgium) followed by mechanical removal of the corona radiate with the use of plastic pipette stripper tips (EZ strip, Research Instruments Ltd, UK) with decreasing inner diameters of 290 and $135 \mu \mathrm{m}$.

Oocytes are assessed for their maturation and for their morphology under an inverted microscope (intgera Ti, R.I., Olympus, IX51/IX70, Tokyo, Japan) at $\times 400$ magnification. Metaphase II oocytes were separated from the immature oocytes (metaphase I oocytes and germinal vesicle) just before sperm injection (3-4 $\mathrm{h}$ after retrieval).

\section{Sperm preparation}

In the case of azoospermia, sperms are retrieved by the urologist, under local anesthesia, either by percutaneous epididymal sperm aspiration (PESA) or testicular sperm aspiration (TESA).

The technical procedure for PESA involved the insertion of a needle attached to a syringe through the scrotal skin into the epididymis. Originally, the use of a larger butterfly needle was described. Currently, most experts use a fine needle (26 gage) attached to a tuberculin syringe containing sperm washing medium. The epididymis is stabilized between the index finger, thumb, and forefinger. After creating negative pressure by pulling the syringe plunger, the tip of the needle is gently and slowly moved in and out of the epididymis until fluid is aspirated. If motile sperm are not obtained, PESA may be repeated at a different site (from the cauda to caput epididymis) until an adequate number of motile sperm is retrieved.

In TESA, the testis is divided into three poles: upper, middle, and lower poles. A needle was inserted through the scrotal skin into the anteromedial or anterolateral portion of the upper pole at an oblique angle toward the medium and lower poles. These aspirations are usually 
carried out using either fine (testicular fine-needle aspiration (TEFNA)) or a scalp butterfly cannula gauge 23 attached to a syringe. The testicular parenchyma is aspirated by creating negative pressure, and the tip of the needle is moved within the testis to disrupt the seminiferous tubules; the specimen was sent to the laboratory for microscopic examination. TESA can be carried out in the contralateral testis if no sperms are obtained during the first attempt.

The obtained tissues from the epididymis or the testis were placed in a special media for handling gametes. By using a scalpel or surgical blade, the tissue was sliced or dissected mechanically, and wet slides prepared were examined under phase contrast microscope (Olympus BX41 Tokyo, Japan) at $\times 200$ magnification. It was then placed in a flushing media for washing. After that, if sperm was found then it is dissected to remove tissues and blood cells, and then the content was placed into an Eppendorf tube and kept in an incubator for $30 \mathrm{~min}$ under $37{ }^{\circ} \mathrm{C}$ then centrifuged for $5-10 \mathrm{~min}$. The supernatant was removed. After adding $1 \mathrm{ml}$ of flushing media, it was then kept in incubator for another $30 \mathrm{~min}$ and centrifuged for 5-10 min. The supernatant was discarded, and the remaining was used as fresh sample for ICSI when done for diagnostic specimens cryopreserved for the next cycle.

\section{Cryopreservation of samples}

After the semen is allowed to be liquefied in the room temperature, it was then mixed with freezing media 1 : $0.7 \mathrm{ml}$ from SpermFreeze ${ }^{\mathrm{TM}}$ (FertiPro) in drops with gentle swirling, then we leave the mixture at room temperature for $10 \mathrm{~min}$ for equilibration. The mixture was put in cryovial then fixed in the freezing straw; leave the straw fixed in a liquid nitrogen vapor for $15 \mathrm{~min}$ then transfer quickly into the liquid nitrogen and store at $196^{\circ} \mathrm{C}$.

\section{Thawing}

We removed as many cryovials as required from the liquid nitrogen and placed the cryovial in tap water for 5 min. We placed the cryovial mixture into the centrifuge tube diluted with sperm wash media (FertiCult TM Flushing medium) of $3 \mathrm{ml}$ per 0.5 sperm mixture mixed thoroughly then centrifuged it for $15 \mathrm{~min}$ at $300-350 \mathrm{~g}$. We resuspended the pellet in sperm wash media for 15 min then used for ICSI.

\section{Intracytoplasmic sperm injection}

Intracytoplasmic sperm injection was performed in MII oocytes according to the technique described by [18]. Oocytes were transferred to the ICSI dish prepared with drops of IVF media (ferticult-IVF, FertiPro N.V., Belgium) covered by mineral oil.

\section{Assessment of fertilization, embryo cleavage, and embryo transfer}

Fertilization assessments were performed $17 \pm 1 \mathrm{~h}$ postinjection. Normally, fertilized oocytes should be spherical and have two polar bodies and two PNs. PNs should be juxtaposed, approximately the same size, and centrally positioned in the cytoplasm with two distinctly clear, visible membranes [23].

Embryo quality was evaluated under an inverted microscope. The following parameters were recorded: (1) the number of blastomeres, (2) the fragmentation percentage, (3) variation in blastomere symmetry, and (4) defects in the zona pellucida and the cytoplasm.

High-quality (grade A) embryos were defined as those having all of the following characteristics: either 4-6 cells on day 2 or $8-10$ cells on day 3 of development, less than $15 \%$ fragmentation, symmetric blastomeres, colorless cytoplasm with moderate granulation with no inclusions, absence of perivitelline space granularity, and absence of zona pellucida dysmorphism. Embryos lacking any of the above characteristics were considered as low quality [24]. For each couple, 1-4 embryos were transferred, depending on the embryo quality and the female's age. Embryo transfer was canceled if no embryos were available. Embryo transfer was performed on day 2 or day 3 using a Gynetics catheter (Gynetics Medical Products N.V, Lommel, Belgium). Transfers were performed with transabdominal ultrasound guidance.

\section{Statistical analysis}

All the statistical analysis procedures are performed using SAS 9.4 statistical software (SAS institute Inc. Cary, NC, USA@ 2014). The differences for continuous variables were examined using Wilcoxon-MannWhitney test for samples without assumed normal distribution. The chi-square test was used for categorical variables and proportions, and for categorical variables with cell counts less than five, Fisher's exact test was used. $p$ values less than 0.05 are considered statistically significant.

Table 1 Comparison between fresh and frozen-thawed obstructive azoospermia group regarding different criteria and outcomes

\begin{tabular}{llll}
\hline Variables & $\begin{array}{l}\text { Fresh OA } \\
\text { Mean }( \pm \text { SD })\end{array}$ & $\begin{array}{l}\text { Frozen OA } \\
\text { Mean }( \pm \text { SD })\end{array}$ & $p$ value \\
\hline Number of patients & 66 & 25 & \\
Age of wife & $31.1( \pm 5.75)$ & $30.7( \pm 6.2)$ & 0.75 \\
Age of husband & $36.6( \pm 8.9)$ & $37( \pm 8.5)$ & 0.69 \\
Number of oocyte & $6.6( \pm 4.2)$ & $8.7( \pm 4.7)$ & 0.04 \\
Number of grade A embryos & $1.38( \pm 1.7)$ & $1.1( \pm 1.2)$ & 0.42 \\
\hline
\end{tabular}


Table 2 Comparison of outcomes between fresh and frozenthawed obstructive azoospermia group

\begin{tabular}{llll}
\hline & Fresh OA \% & Frozen OA \% & $p$ value \\
\hline Fertilization rate & 57 & 47 & 0.093 \\
Clinical pregnancy & 23.7 & 17.4 & 0.54 \\
Live birth & 11.9 & 8.7 & 0.68 \\
\hline
\end{tabular}

\section{Results}

In the obstructive azoospermia (OA) group which has 91 cases, 66 of fresh spermatozoa were used for ICSI and 25 frozen-thawed spermatozoa were used for ICSI in which the result shows no significant difference in age of wife and husband and grade A embryos and significant difference in number of oocyte retrieved as shown in Table 1.

The fertilization rate, clinical pregnancy rate, and live birth rate show no significant differences, as shown in Table 2.

In the non-obstructive azoospermia (NOA) group, we had 68 cases; in 32 of them, fresh spermatozoa were used for ICSI, and in 36 of them, frozen-thawed spermatozoa were used for ICSI, and there was no significant difference regarding the age of couple, number of retrieved oocyte, and grade A embryos as shown in Table 3.

Clinical pregnancy were recorded from five cases using fresh spermatozoa, but only one of them reached full term, as from four clinical pregnancies from the group who used frozen-thawed spermatozoa, one reached full term. Fertilization rate, clinical pregnancy, and live birth rate show no significant differences as shown in Table 4.

\section{Discussion}

After reporting the first ICSI trial with frozen testicular sperm, the advantages of cryopreserving testicular sperm for ICSI are well known [25, 26].

The main advantages of using frozen-thawed sperm for ICSI are to avoid repeated surgical biopsy and avoid useless ovarian stimulation.

However, there are several drawbacks of using frozenthawed sperm for ICSI; first, there is a substantial risk of

Table 3 Comparison between fresh and frozen-thawed nonobstructive azoospermia group regarding different criteria and outcomes

\begin{tabular}{llll}
\hline Variables & $\begin{array}{l}\text { Fresh NOA } \\
\text { Mean }( \pm \text { SD) }\end{array}$ & $\begin{array}{l}\text { Frozen NOA } \\
\text { Mean }( \pm \text { SD) }\end{array}$ & $p$ value \\
\hline Number of patients & 32 & 36 & \\
Age of wife & $32.8( \pm 5.8)$ & $30.5( \pm 7)$ & 0.14 \\
Age of husband & $36.5( \pm 6.6)$ & $36.1( \pm 7.35)$ & 0.98 \\
Number of oocyte & $5.6( \pm 3.1)$ & $5.2( \pm 3.3)$ & 0.61 \\
Number of grade A embryos & $0.34( \pm 0.6)$ & $0.42( \pm 0.73)$ & 0.66 \\
\hline
\end{tabular}

Table 4 Comparison of outcomes between fresh and frozenthawed non-obstructive azoospermia group

\begin{tabular}{llll}
\hline & Fresh NOA \% & Frozen NOA \% & $p$ value \\
\hline Fertilization rate & 37 & 36 & 0.91 \\
Clinical pregnancy & 20 & 14.3 & 0.58 \\
Live birth & 4 & 3.6 & 0.93 \\
\hline
\end{tabular}

$\sim 20 \%$ of not finding sperm suitable for injection despite extreme efforts in the case of NOA.

Second is regarding motility of the sperm as we know it is an indicator for sperm viability, although in vitro culture of frozen-thawed testicular sperm improve the motility in OA but not so promising in the case of NOA where the only immotile sperm was found $1011 \mathrm{MX}$.

In the case of OA, our study shows no significant difference when we compared fresh sperm versus frozenthawed sperm regarding fertilization rate (57\%, 47\%), clinical pregnancy rate $(23.7 \%, 17.4 \%)$, and live birth rate $(11.9 \%, 8.7 \%)$ as shown in previous studies [18, 27, 28].

Regarding NOA, the fertilization rate is nearly the same for fresh and frozen-thawed which was 37\% and $36 \%$ respectively with no significant difference.

Also, there are no significant differences in clinical pregnancy rate and live birth rate $(5 \%, 4 \%, p$ value 0.71$)$ and $(1 \%, 1 \%, p$ value $=1)$ respectively.

Our results similar to $[18,27-30]$, but unlike [31] which show improved embryo quality, there is higher pregnancy rate and lower abortion rate, and also, Mohamed and Shedeed (2008) show improved pregnancy rate in the fresh sperms in NOA [32].

The limitation in our study was due to difficulty in data collection due to missing information, which leads to small sample size, which may affect the significance of our results.

In conclusion, cryopreservation of testicular sperm is a reliable technique to do before ovulation induction especially in cases of non-obstructive azoospermia.

\section{Conclusion}

Cryopreservation of testicular sperm is reliable if carried out before ovulation induction especially in cases with non-obstructive azoospermia.

Abbreviations

ICSI: Intracytoplasmic injection; NOA: Non-obstructive azoospermia; OA: Obstructive azoospermia; SD: Standard deviation

Acknowledgements

We acknowledge all staffs who worked in Dwarozh IVF Center.

Authors' contributions

The author read and approved the final manuscript.

Funding

No funding 


\section{Availability of data and materials}

The data that support the findings of this study are available from IVF center, but restrictions apply to the availability of these data.

\section{Ethics approval and consent to participate}

Informed consent was taken from all patients. Ethical approval from the ethics committee in Sulaimani University College of Medicine was obtained and are available upon request to upload.

\section{Consent for publication}

Not applicable

\section{Competing interests}

The author declares that he/she has no competing interests.

Received: 29 June 2019 Accepted: 11 November 2019

Published online: 21 December 2019

\section{References}

1. Craft I, Bennett V, Nicholson N (1993) Fertilizing ability of testicular spermatozoa. Lancet 342:864

2. Schoysman R, Vanderzwalmen P, Nijs M, Segal-Bertin G, van de Casseye M (1993) Successful fertilization by testicular spermatozoa in an in-vitro fertilization programme. Hum Reprod. 8(8):1339-1340

3. Aziz N, Agarwal A, Nallella KP, Thomas AJ (2006) Jr Relationship between epidemiological features and aetiology of male infertility as diagnosed by a comprehensive infertility service provider. Reprod Biomed. 12(2):209-214

4. Jequier AM (1985) Obstructive azoospermia: a study of 102 patients. Clin Reprod Fertil. 3(1):21-36

5. Jarow JP, Espeland MA, Lipshultz LI (1989) Evaluation of the azoospermic patient. J Urol. 142(1):62-65

6. Nagy Z, Liu J, Cecile J, Silver S, Devroey P, Van Steirteghem A (1995 Apr) Using ejaculated, fresh, and frozen-thawed epididymal and testicular spermatozoa gives rise to comparable results after intracytoplasmic injection. Fertil Steril. 63(4):808-815

7. Fahmy I, Mansour R, Aboulghar M, Serour G, Kamal A, Tawab NA et al (1997) Intracytoplasmic sperm injection using surgically retrieved epididymal and testicular spermatozoa in cases of obstructive and nonobstructive azospermia. Int j Androl. 20:37-40

8. De Croo I, Van der Elst J, Everaert K, De Sutter P, Dhont M (2000 Jun) Fertilization, pregnancy and embryo implantation rates after ICSI in cases of obstructive and non-obstructive azoospermia. Hum Reprod. 15(6):1383-1388

9. Tournaye $H$, Camus M, Vandervorst M, Nagy Z, Joris H, Van Steirteghem A, Devroey P (1997) Surgical sperm retrieval for intracytoplasmic sperm injection. Int J Androl. 20(suppl 3):69-73

10. Palermo GD, Schlegel PN, Hariprashad JJ, Ergun B, Mielnik A, Zaninovic N. Fertilization and pregnancy outcome with intracytoplasmic sperm injection for azoospermic men. 1999:14:741-8.

11. Balaban B, Urman B, Isiklar A, Alatas C, Mercan R (2001) Aksoy S and nouhoglu A. Blastocyst transfer following intracytoplasmic injection of ejaculated, epididymal or testicular spermatozoa. Hum Reprod. 16:125-129

12. Bukulmez O, Yucel A, Yarali H, Bildirici I, Gurgan T (2001) The origin of spermatozoa does not affect intracytoplasmic sperm injection outcome. Eur J Obstet Gynecol Reprod Biol. 94:250-255

13. Sousa M, Cremades N, Silva J, Oliveira C, Ferraz L, Teixeira da silva J, Viana P, Barros A (2002) Predictive value of testicular histology in secretary azospermic subgroups and clinical outcome after microinjection of fresh and frozen-thawed sperm and spermatids. Hum Reprod 17:1800-1810

14. Schlegel PN, Su LM (1997) Physiological consequences of testicular sperm extraction. Hum Reprod. 12(1):1688-1692

15. Ron-el R, Strauss S, Freidler S, Strassburger D, Komarovsky D, Raziel A (1998) Serial sonography and color flow Doppler imaging following testicular and epididymal sperm extraction. Hum Reprod. 13:3390-3393

16. Windt ML, Coetzee K, Kruger TF, Menkveld R, Van der Merwe JP (2002 Feb) Intracytoplasmic sperm injection with testicular spermatozoa in men with azoospermia. J Assist Reprod Genet. 19(2):53-59

17. Tournaye H, Merdad T, Silber S et al (1999) No differences in outcome after intracytoplsmic sperm injection with fresh or with frozen-thawed epididymal spermatozoa. Hum Reprod. 14:90-95
18. Habermann H, Seo R, Cieslak J, Niederberger C, Prins GS, Ross L (2000 May) In vitro fertilization outcomes after intracytoplasmic sperm injection with fresh or frozen-thawed testicular spermatozoa. Fertil Steril. 73(5):955-960

19. Wood S, Thomas K, Schnauffer K, Troup S, Kingsland C, Lewis-Jones I (2002) Reproductive potential of fresh and cryopreserved spermatozoa epididymal and testicuar in consecutive intracytoplasmic sperm injection cycles in the same patients. Fertil Steril 77:1162-1166

20. Christodoulou K, Jerkovic S, Geyer J, Allen L, Tindall L, Mantoudis E, Croft IL (2002) The effect of TESA cryopreservation on the outcome of ICSI cycles. BFS/ACE abstracts. Hum Fertil 5:86

21. De Croo I, Van der Elst J, Everaert K, De Sutter P, Dhont M (1998) Fertilization, pregnancy and embryo implantation rates after ICSI with fresh or frozen-thawed testicular spermatozoa. Hum Reprod. 13:1893-1897

22. Verheyen G, De Croo I, Tournaye H, Pletincx I, Devroey P, Van AC (1995) Comparison of four mechanical methods to retrieve spermatozoa from testicular tissue. Hum Reprod. 10:2956-2959

23. Brooks AK, Jeffrey VM, Christopher JDJ (2000) Hand book of the assisted reproduction laboratory, micromanipulation of human gametes, zygotes and embryos. Second eds. USA.CRC.:223-227

24. Papale L, Fiorentino A, Montag M, Tomasi G (2012) The zygote. Hum. Reprod. 27(S1):i22-i49

25. Hovatta O, Foudila T, Siegberg R, Johansson Kand Reima I (2004) Pregnancy resulting from intracytoplasmic injection of spermatozoa from afrozenthawed testicular biopsyspecimen. Hum Reprod. 19(12):2822-2830

26. Romero J, Remohi J, Minguez Y, Rubio C, Pellicer A, Gil-Salom M (1996) Fertilization after intracytoplasmic sperm injection with cryopreserved testicular spermatozoa. Fertil Steril. 65:877-879

27. Fukunaga N, Haigo K, Araki Y (2001) Efficiency of using frozen-thawed testicular sperm for multiple intracytoplasmic sperm injection. J Assist Reprod Gene. 18(12):634-637

28. Wu B, Wong D, Lu S, Dickstein S, Silva M, Gelety TJ (2005 Dec) Optimal use of fresh and frozen-thawed testicular sperm for intracytoplasmic sperm injection in azoospermic patients. J Assist Reprod Genet. 22(11-12):389-394

29. Tavukcuoglu S, AL-Azawi T, Al-Hasani S, Afshin Khaki A, Khaki A, Tasdemir S (2018) 2013;14(2):79-84. Frozen-thawed testicular sperm in men with nonobstructive azoospermia: asystematic review and metaanalysis. J Assist Reprod Genet. 35(7):1247-1257

30. Yuz Z, Wei Z, Yang J, Wang T, Jiang H, Li H et al (2018) Comparison of intracytoplasmic sperm injection outcome with fresh versus frozen-thawed testicular sperm in men with nonobstructive azoospermia: a systematic review and metaanalysis. J Assist Reprod Genet 35(7):1247-1257

31. Aoki W, Wilcox AL, Throrp C, Hamilton BD, Carrell DT (2004) Improved in vitro fertilization embryo quality and pregnancy rates with intracytoplasmic sperm injection of sperm from fresh testicular biopsy samples versus frozen biopsy samples. Fertil Seteril. 82(6):1532-1535

32. Moustapha MK (2008) and ashour S. Fresh versus cryopreserved testicular spermatozoa in men with nonobstructive azoospermia. Middle East Fertility Society J 13(1):59-62

\section{Publisher's Note}

Springer Nature remains neutral with regard to jurisdictional claims in published maps and institutional affiliations.

\section{Submit your manuscript to a SpringerOpen ${ }^{\circ}$ journal and benefit from:}

- Convenient online submission

- Rigorous peer review

- Open access: articles freely available online

- High visibility within the field

- Retaining the copyright to your article

Submit your next manuscript at $>$ springeropen.com 